\title{
Diferencias regionales en la mortalidad por cáncer de mama y cérvix en México entre 1979 y 2006
}

\author{
Lina Sofía Palacio-Mejía, D en Pobl,(1) Eduardo Lazcano-Ponce, D en C,(2) \\ Betania Allen-Leigh, D en Antrop, ${ }^{(3)}$ Mauricio Hernández-Ávila, D en C. ${ }^{(4)}$
}

\author{
Palacio-Mejía LS, Lazcano-Ponce E, \\ Allen-Leigh B, Hernández-Ávila M. \\ Diferencias regionales en la mortalidad por cáncer \\ de mama y cérvix en México entre 1979 y 2006. \\ Salud Publica Mex 2009;5 I supl 2:S208-S219.
}

\section{Resumen}

Objetivo. Explorar las diferencias regionales en la mortalidad por cáncer de mama $(\mathrm{CaMa})$ y cervical $(\mathrm{CaCu})$ en México. Material y métodos. Se calcularon tendencias de mortalidad por $\mathrm{CaMa}$ y $\mathrm{CaCu}$ mediante modelos probabilísticos ajustados por estado, grado de marginación y lugar de residencia (urbano/rural). Resultados. La tendencia de mortalidad por CaMa ha sido ascendente, de una tasa estandarizada de 5.6 muertes por cada 100000 mujeres en 1979 a I0.I en 2006. La mortalidad por $\mathrm{CaCu}$ alcanzó un pico en 1989 y a partir de esa fecha se redujo a 9.9 en 2006. Las tasas más altas de mortalidad por CaMa se encuentran en la capital (I3.2) y la región norte (II.8), mientras en el sur se registra la mortalidad por $\mathrm{CaCu}$ más alta (I I.9). Discusión. El número de muertes por $\mathrm{CaMa}$ aumenta de forma gradual a lo largo del tiempo a nivel nacional y persisten elevadas tasas de mortalidad por $\mathrm{CaCu}$ en áreas marginadas.

Palabras clave: cáncer de mama; cáncer cervical; tasas de mortalidad; diferencias regionales; riesgo relativo; México
Palacio-Mejía LS, Lazcano-Ponce E, Allen-Leigh B, Hernández-Ávila M.

Regional differences in breast and cervical cancer mortality in Mexico between 1979-2006.

Salud Publica Mex 2009;5I suppl 2:S208-S219.

\section{Abstract}

Objective. Explore the regional differences in breast (BC) and cervical cancer (CC) mortality in Mexico. Material and Methods. We estimated mortality trends for $\mathrm{BC}$ and $\mathrm{CC}$ using probabilistic models adjusted by state marginalization level and urban and rural residence. Results. BC mortality shows a rising trend, from a rate of 5.6 deaths per 100000 women in 1979 to 10.1 in 2006. The CC mortality rate reached a peak in 1989 and after this decreased significantly to 9.9 in 2006. The highest BC mortality rates are found in Mexico City (I3.2) and the northern part of the country (II.8). As for $\mathrm{CC}$, the highest mortality rates are found in the south (II.9 per 100000 women the). Discussion. The number of $\mathrm{BC}$ cases are increased gradually at the national level during the last three decades and high rates of CC mortality persist in marginalized areas.

Key words: breast cancer; cervical cancer; mortality rates; regional differences; relative risk; Mexico

(I) Dirección de Enfermedades Crónicas, Centro de Investigaciones en Salud Poblacional, Instituto Nacional de Salud Pública (INSP), México.

(2) Centro de Investigaciones en Salud Poblacional, INSP, México.

(3) Dirección de Salud Reproductiva, Centro de Investigaciones en Salud Poblacional, INSP, México.

(4) Subsecretaría de Prevención y Control de Enfermedades. Secretaría de Salud. México, DF. 
$\mathrm{L}$ a prioridad de los sistemas de salud desarrollados es que toda la población, cualesquiera que sean su condición socioeconómica y el grupo étnico al que pertenezca, tenga las mismas oportunidades y calidad en la accesibilidad y servicios de atención preventiva y curativa. ${ }^{1}$ Diversos estudios, ${ }^{2}$ revisiones sistemáticas ${ }^{3-5}$ y metaanálisis ${ }^{6}$ han documentado las disparidades que existen en relación con la incidencia, cobertura de tamizaje, tratamiento, sobrevida y mortalidad por cáncer en la mujer, en especial en países de ingresos altos. ${ }^{7}$ La evidencia muestra que poblaciones marginadas, en términos sociales, geográficos y económicos, tienen una mayor probabilidad de morir por cánceres prevenibles, lo cual se puede atribuir entre otros factores a que no reciben un diagnóstico oportuno y por tanto el tratamiento es tardío. ${ }^{1,8}$ En México se registra una elevada incidencia y mortalidad por cáncer de mama $(\mathrm{CaMa})^{9}$ y cérvix, $(\mathrm{CaCu})^{10,11}$ fenómeno que surge de las disparidades sociales y a su vez las propicia. En el resto del mundo se ha documentado que las desigualdades vinculadas con estos dos cánceres ginecológicos existen vinculados con el lugar de residencia, grupo étnico, estado socioeconómico y acceso a la seguridad social, entre otros factores. ${ }^{12,13} \mathrm{Sin}$ embargo, hay significativamente menos investigación y análisis sobre posibles disparidades respecto del cáncer en países de ingresos medios y bajos, ${ }^{14}$ como es el caso de México.

La exploración de las posibles inequidades, en cuanto a la epidemiología, alternativas de tamizaje, calidad del tratamiento, sobrevida y mortalidad referentes al cáncer de mama y cervical en México, es importante para lograr mayor eficiencia en términos del costo y lograr un impacto más equitativo de los recursos destinados a la prevención primaria y secundaria de estas neoplasias. Son necesarios la recolección y el análisis de datos cuantitativos y cualitativos para lograr una mejor comprensión de los factores que contribuyen a estas disparidades e idear posibles medidas para disminuirlas. ${ }^{715}$ Por esta razón, el objetivo del presente artículo es describir la forma en que se distribuye la mortalidad por cáncer de mama y cáncer cervical entre las mujeres mexicanas, con objeto de conocer las potenciales disparidades regionales en los diferentes estados de la República Mexicana, además de estratificar por áreas rurales y urbanas; en última instancia, deben cuantificarse las diferencias regionales en la mortalidad por cáncer de mama en México, así como indicar si la mortalidad por cáncer cervical sigue concentrada en áreas rurales, como se ha constatado en estudios anteriores. ${ }^{16}$

\section{Material y métodos}

En el análisis de la mortalidad por cáncer cervical y cán- cer de mama se utilizaron las tasas brutas de mortalidad y las tasas estandarizadas para la población mundial. Para el cálculo de las tasas brutas de mortalidad se emplearon como fuentes de información las estadísticas vitales de mortalidad publicadas por el Instituto Nacional de Estadística, Geografía e Informática (INEGI) y la Secretaría de Salud de México de los años de 1979 a $2006,{ }_{1}^{17}$ y como denominador se usaron las proyecciones de población de 1970 a 2030 que realizó el Consejo Nacional de Población (CONAPO). ${ }^{18}$ Para estandarizar las tasas brutas, se utilizó la estructura poblacional mundial elaborada por las Naciones Unidas. ${ }^{19}$ Estas tasas se calcularon por entidad federativa y región.

Una manera de señalar las desigualdades (de desarrollo, socioeconómicas y geográficas) consiste en emplear la clasificación regional. Para la regionalización del país se retomó el Plan Nacional de Desarrollo 2007-2012, ${ }^{20}$ el cual divide las 32 entidades de México en tres subregiones: norte, centro y sur. Con posterioridad se extrajo al Distrito Federal dela región centro y se lo tomó como una cuarta región, en virtud de la concentración de servicios de salud en la ciudad y su bajo índice de marginación.

La región norte se constituyó con los estados de Baja California, Baja California Sur, Chihuahua, Coahuila, Durango, Nuevo León, San Luis Potosí, Sinaloa, Sonora, Tamaulipas y Zacatecas. La región centro se integró con Aguascalientes, Colima, Guanajuato, Hidalgo, Jalisco, Estado de México, Michoacán, Morelos, Nayarit, Puebla, Querétaro y Tlaxcala. Por último, la región sur incluyó a Campeche, Chiapas, Guerrero, Oaxaca, Quintana Roo, Tabasco, Veracruz y Yucatán.

El cálculo de los valores regionales para todos los indicadores e índices seleccionados se realizó con base en la disponibilidad de la información original. En el caso de la mortalidad por cáncer cervical y mamario, los valores regionales se calcularon a partir de la información original, con base en los siguientes procedimientos:

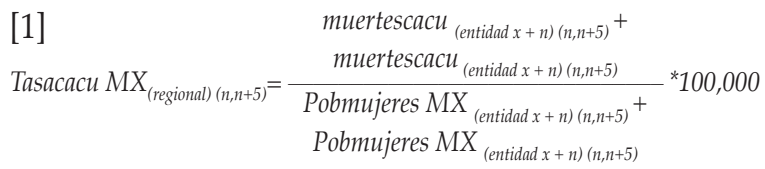

donde $\mathrm{n}$ es el número de estados que componen cada región

[2]

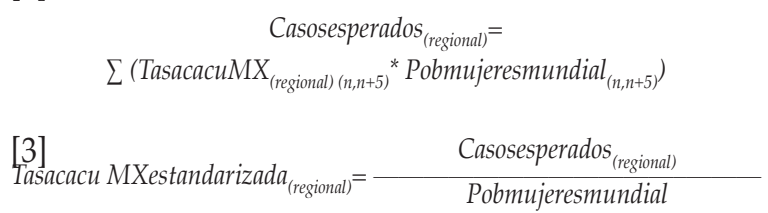




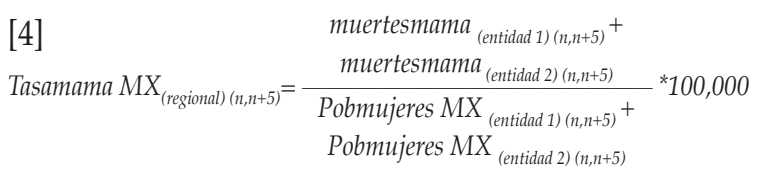

[5]

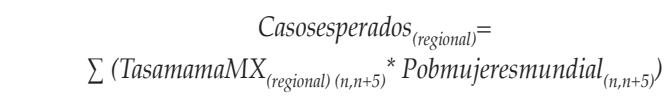

Tasamamaestandarizada $_{(\text {(regional) }}=\frac{\text { Casosesperados }_{(\text {(regional) }}}{\text { Pobmujeresmundial }}$

Se retomó el índice de marginación elaborado por CONAPO, el cual es una medida-resumen que permite diferenciar los estados y municipios del país, según sean el impacto global de las carencias que padece la población como resultado de la falta de acceso a la educación, la residencia en viviendas inadecuadas, la percepción de ingresos monetarios insuficientes y las relacionadas con la residencia en localidades pequeñas. El índice de marginación es una herramienta que contribuye a formular diagnósticos exhaustivos, identificar las disparidades socioespaciales que persisten en los estados y municipios del país y, con ello, apoyar el diseño e instrumentación de programas y acciones dirigidos a fortalecer la justicia distributiva en el ámbito regional y la atención prioritaria de la población con mayor desventaja. ${ }^{21}$

Se estimaron los riesgos relativos de los indicadores de mortalidad por cáncer cervical y cáncer de mama, a través de una regresión de Poisson, para las entidades federativas y estratificadas por tipo de residencia, ya sea urbana o rural. Se construyeron a partir de las tasas de mortalidad calculadas a nivel de localidad para poder contar con indicadores por estrato urbano y rural. Para clasificar las localidades como rurales o urbanas se usó el punto de corte propuesto por el INEGI de 2500 habitantes (una localidad con 2500 habitantes o más se califica como urbana y cuando tiene menos de este número de habitantes se clasifica como rural). ${ }^{22}$

La regresión de Poisson se utiliza para comparar las tasas de mortalidad por una enfermedad, ajustadas por entidad federativa y población urbana - rural, para lo cual se emplean como referencia de comparación las tasas de mortalidad del Distrito Federal, el cual posee el nivel más alto de desarrollo económico en el país respecto de los 31 estados. De igual modo, este tipo de regresión permite observar las diferencias regionales del riesgo de morir por cada causa.
[7]

$$
\log (E(y))=\beta 0+\beta 1 \text { arearur }- \text { urb }+\beta 2 \text { entidadfederativa }
$$

La regresión de Poisson se usa con distribuciones no lineales como la que presenta el crecimiento poblacional y sirve para estudiar sucesos de baja ocurrencia, en los cuales se cuenta con un conteo de casos, tras asumir que tales sucesos son independientes. Para este modelo se tomó como variable dependiente el número de muertes por cáncer cervical o cáncer de mama y el conjunto de variables independientes fueron la entidad federativa y la población urbana-rural, con el fin de buscar variaciones del riesgo de morir por cáncer cervical en la población femenina.

[8]

RRcacu $=\frac{\text { Casosdecacu }_{\left(\text {Pob1 }_{1}\right)} / \text { TamañodelaPob1 }}{\text { Casosdecacu }_{\left(\text {Pobreferencia }_{)}\right.} / \text {TamañodelaPobreferencia }}$

donde, si $\mathrm{RR}=1$, el riesgo relativo de la población de referencia es semejante al de la otra población; si RR < 1 , el riesgo de la población 1 es menor que el de la población de referencia; $\mathrm{si} R \mathrm{R}>1$, el riesgo de la población 1 es mayor que el de la población de referencia.

\section{Resultados}

\section{Número de defunciones y tasas de mortalidad por cáncer de mama y cáncer cervical (1979-2006)}

El número de defunciones por cáncer cervical empieza a ascender en 1979 hasta presentar una meseta a fines del decenio de 1990 y mostrar un ligero descenso después del año 2000 (figura 1). En el caso del cáncer de mama, el número de defunciones comienza a un nivel mucho menor, pero asciende de manera constante hasta superar el número de muertes por cáncer cervical al final del periodo estudiado (figura 1). En relación con las tasas de mortalidad, la tendencia de mortalidad por cáncer de mama ha sido ascendente durante todo el periodo observado, desde una tasa estandarizada de 5.6 mujeres fallecidas por cada 100000 mujeres en 1979 hasta una tasa estandarizada de 10.1 en 2006 (figura 2).

La evolución de la mortalidad por cáncer cervical tiene una tendencia ascendente desde 1979 hasta 1989, año en que alcanza la tasa estandarizada más alta durante el periodo de 1979 a 2006, con 16.2 mujeres fallecidas por cada 100000 mujeres. Es sólo a partir de 1990 que comienzan a disminuir las tasas de mortalidad por cáncer cervical de forma persistente hasta alcanzar en el año 2006 una tasa de 9.9 (figura 2). 


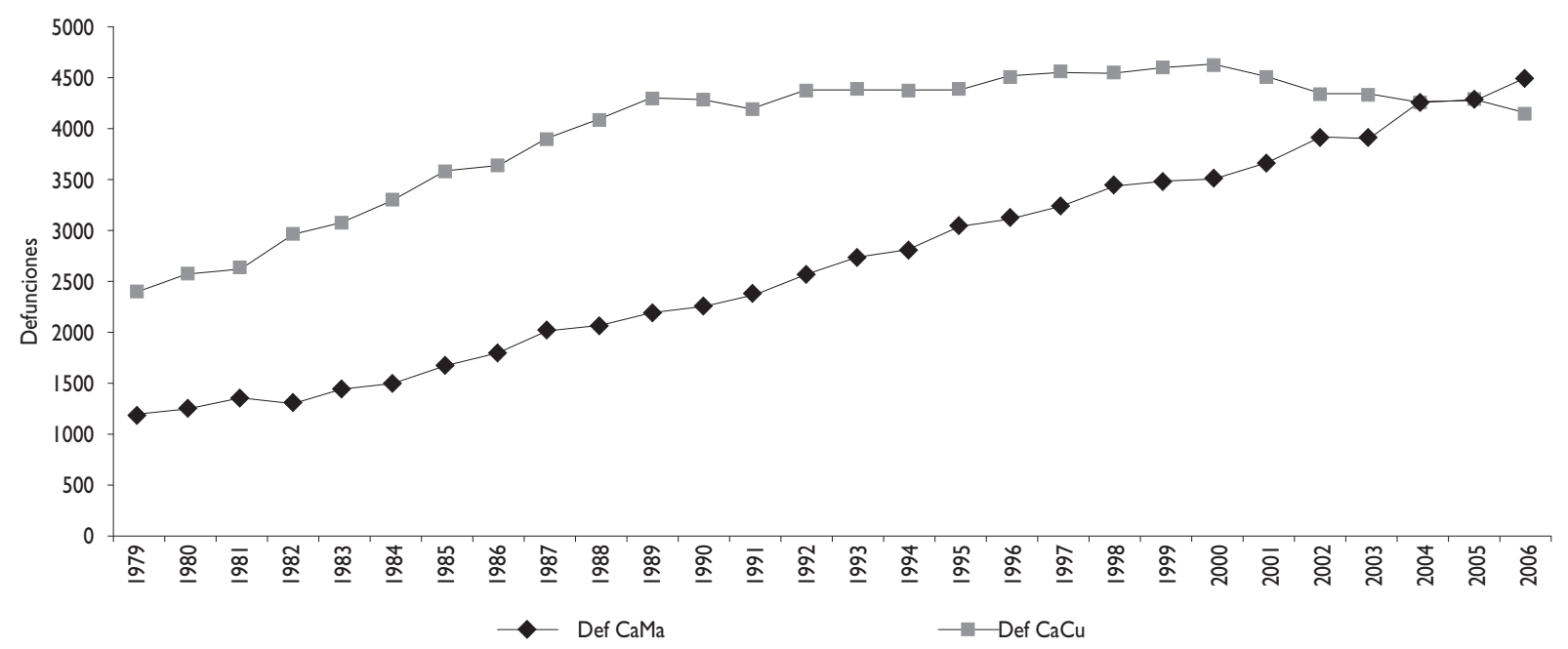

Fuente: Estadísticas vitales de mortalidad 1979 a 2006 INEGI/SSA

Figura I. Número de defunciones de por cáncer CerVical y CÁnCer de mama, MéXico, 1979 a 2006

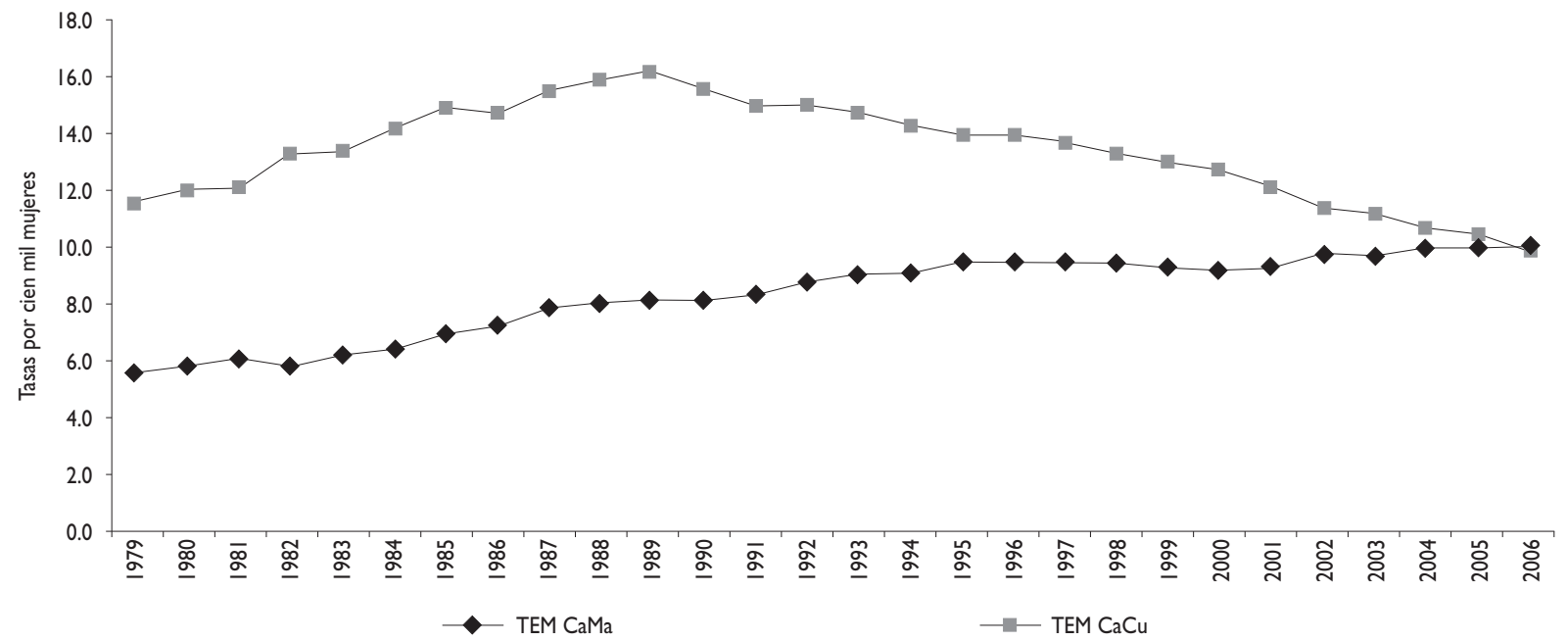

Fuente: Estadísticas vitales de mortalidad 1979 a 2006 INEGI/SSA. Proyecciones de población 1970 a 2030 CONAPO

División de Población del Departamento de Asuntos Sociales y Económicos de la Secretaría de Naciones Unidas, Perspectivas de la Población Mundial: Revisión 2004 y Perspectivas de la Urbanización Mundial: Revisión 2003, http://esa.un.org/unpp, 24 de Febrero 2006

TEM CaCu:Tasa estandarizada de mortalidad por cáncer cervical por cien mil mujeres

TEM CaMa:Tasa estandarizada de mortalidad por cáncer de mama por cien mil mujeres

Figura 2.TASAs estandarizadas de MORTalidad POR CÁNCer CerVICAL Y CÁNCer de MAMA. MÉXICO, 1979 a 2006

Al comparar la evolución de las tasas de mortalidad por cáncer de mama con las tasas de mortalidad por cáncer cervical se observa que a partir del año 2006 estas dos causas cambian de lugar en la frecuencia de la mortalidad en las mujeres. Es decir, hasta el año 2004 la mortalidad por cáncer cervical era la principal causa de mortalidad, mientras que el cáncer de mama era la segunda causa y en el año 2006 este último se convierte 
en la primera causa de mortalidad por cáncer en mujeres en edad reproductiva (figura 2). El número de muertes por cáncer de mama se ha incrementado de manera anual; para el año 1979 existieron 1144 muertes, pero en el 2006 se registraron 4497 casos. Por otro lado, el número de muertes por cáncer cervical en México empieza a disminuir a partir del año 2001, pero aún es preocupante con 4134 mujeres fallecidas en el año 2006.

\section{Distribución regional de tasas de mortalidad por cáncer de mama y cervical}

En cuanto a la distribución geográfica por región de las tasas estandarizadas de mortalidad por cáncer mamario en México para el año 2006, se advierte que las tasas regionales más altas proceden del Distrito Federal (13.2) y la región norte del país (11.8) (figura 3). Sin embargo, el Distrito Federal presenta una disminución de la tasa estandarizada de esta causa de muerte entre los años 2000 y 2006, en tanto que las regiones norte, centro y sur tienen el mismo comportamiento ascendente que presenta el total nacional. Entre las entidades que conforman la región norte, resalta la tasa del estado de Zacatecas por ser la más baja (6.4). En la región centro, con un promedio de 9.70 en 2006, se reconoce que es más heterogénea en el comportamiento de las cifras de las entidades federativas que la componen; al respecto, Puebla posee la tasa más baja del centro (6.8) y Jalisco la más alta (14.6). El sur tiene un promedio regional de 7 y

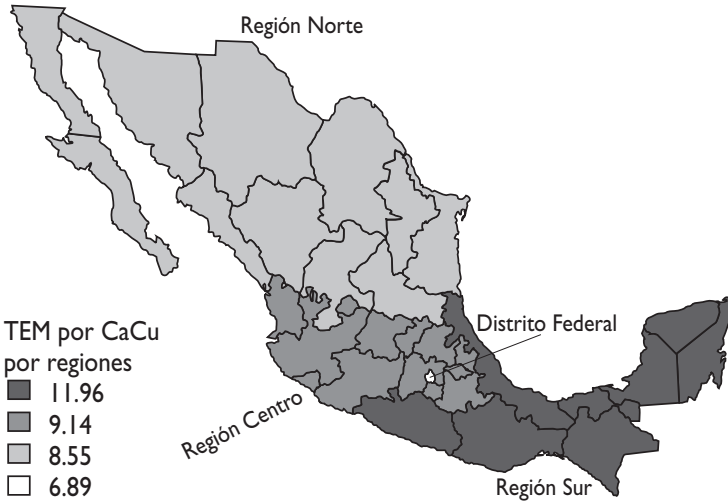

sobresale Oaxaca con la tasa más baja (5.9) y Campeche con la más alta (10.3) (figura 3).

El Distrito Federal, la región norte y la región centro tienen cifras menores de mortalidad por cáncer cervical respecto de las del promedio nacional. Por el contrario, la región sur es donde se registran las tasas más elevadas de mortalidad por cáncer cervical (11.9). Por otra parte, las tasas regionales de mortalidad por cáncer cervical muestran una tendencia a la disminución en el plano nacional. Aunque la disminución es muy homogénea, la región que muestra una reducción mayor es la región centro con-2.9. El Distrito Federal es la región que posee las tasas estandarizadas de cáncer cervical más bajas para el año 2006 (6.9), en comparación con la región norte (8.6) y la región centro (9.1) (figura 3).

En cuanto a la distribución regional, estas dos causas de mortalidad tienen un comportamiento inverso: mientras que el cáncer de mama muestra unas tendencias ascendentes y es mayor en la región norte y el Distrito Federal, la mortalidad por cáncer cervical tiende a disminuir y es mayor en las regiones centro y sur del país.

\section{Riesgo relativo de morir por cáncer de mama y cervical de acuerdo con el área rural o urbana de residencia}

En el cuadro I se presentan los riesgos relativos de mortalidad por cáncer de mama ajustados por estrato urbano-rural y entidad federativa para los años $2000 \mathrm{y}$

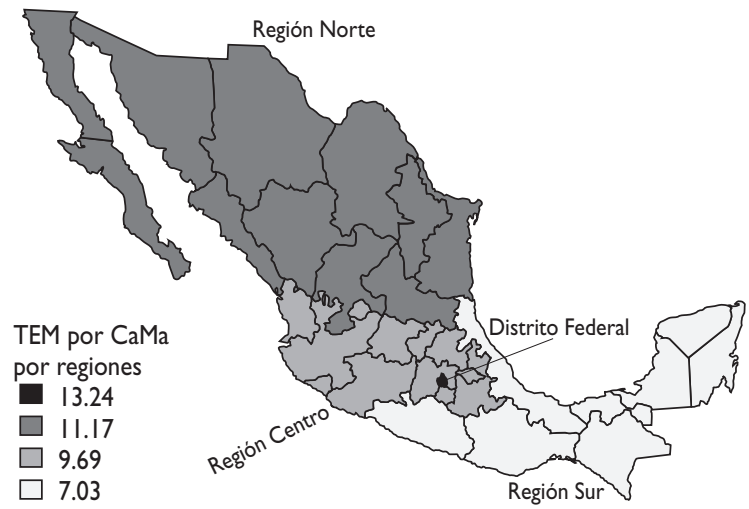

Fuente: Estadísticas vitales de mortalidad 1979 a 2006 INEGI/SSA. Proyecciones de población 1970 a 2030 CONAPO

División de Población del Departamento de Asuntos Sociales y Económicos de la Secretaría de Naciones Unidas, Perspectivas de la Población Mundial: Revisión 2004 y Perspectivas de la Urbanización Mundial: Revisión 2003, http://esa.un.org/unpp, 24 de Febrero 2006

TEM CaCu:Tasa Estandarizada de mortalidad por cáncer cervical por cien mil mujeres

TEM CaMa:Tasa Estandarizada de mortalidad por cáncer de mama por cien mil mujeres

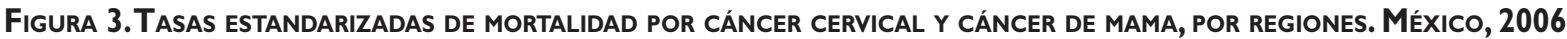


Cuadro I

RIESGO DE MORIR POR CÁNCER DE MAMA POR ENTIDAD FEDERATIVA SEGÚN ESTRATO URBANO-RURAL. México 2000 y 2006

\begin{tabular}{|c|c|c|c|c|c|c|c|}
\hline Entidad & IRR & $\begin{array}{c}2000 \\
1 C \\
95 \%\end{array}$ & & IRR & $\begin{array}{c}2006 \\
1 C \\
95 \%\end{array}$ & & $\begin{array}{l}\text { Diferencias } \\
\text { IRR de Mama } \\
\text { 2000-2006 }\end{array}$ \\
\hline Área (Nacional) & 2.33 & 2.16 & 2.51 & 1.88 & 1.76 & 2.00 & -0.45 \\
\hline Región Norte & 1.07 & 0.99 & 1.15 & 1.14 & 1.07 & 1.21 & +0.07 \\
\hline Baja California & 0.91 & 0.64 & 1.30 & 1 & 0.84 & 1.19 & +0.09 \\
\hline Baja California Sur & 1.19 & 0.70 & 2.01 & 1.43 & 1.01 & 2.02 & +0.24 \\
\hline Coahuila & 0.43 & 0.29 & 0.64 & 1.08 & 0.91 & 1.30 & +0.65 \\
\hline Chihuahua & 0.74 & 0.42 & 1.30 & 1.39 & 1.20 & 1.60 & +0.65 \\
\hline Durango & 0.94 & 0.63 & 1.41 & 1.05 & 0.82 & 1.34 & +0.11 \\
\hline Nuevo León & 1.11 & 0.72 & 1.71 & 1.21 & 1.06 & 1.38 & +0.10 \\
\hline San Luis Potosí & 0.42 & 0.23 & 0.76 & 0.96 & 0.78 & 1.18 & +0.54 \\
\hline Sinaloa & 0.69 & 0.47 & 1.02 & 1.18 & 0.99 & 1.41 & +0.49 \\
\hline Sonora & 1 & 0.70 & 1.42 & 1.11 & 0.93 & 1.34 & +0.11 \\
\hline Tamaulipas & 0.61 & 0.39 & 0.93 & 1.22 & 1.04 & 1.42 & +0.61 \\
\hline Zacatecas & 0.78 & 0.52 & 1.16 & 0.72 & 0.53 & 1.00 & -0.06 \\
\hline
\end{tabular}

$\begin{array}{llllllll}\text { Región Centro } & \mathbf{0 . 8 8} & 0.83 & 0.94 & 0.94 & 0.89 & 0.99 & \mathbf{+ 0 . 0 6}\end{array}$

$\begin{array}{lllllllll}\text { Aguascalientes } & 0.92 & 0.59 & 1.42 & 0.77 & 0.55 & 1.06 & -0.15\end{array}$

$\begin{array}{llllllll}\text { Colima } & 1.23 & 0.88 & \text { I.7I } & 1.23 & 0.87 & 1.74 & +0.00\end{array}$

$\begin{array}{llllllll}\text { Guanajuato } & 0.7 & 0.50 & 0.99 & 0.93 & 0.81 & 1.08 & +0.23\end{array}$

$\begin{array}{llllllll}\text { Hidalgo } & 0.85 & 0.58 & 1.24 & 0.88 & 0.71 & \text { I.10 } & +0.03\end{array}$

$\begin{array}{llllllll}\text { Jalisco } & 1.09 & 0.80 & 1.50 & 1.39 & 1.26 & 1.54 & +0.30\end{array}$

$\begin{array}{lllllllll}\text { México } & 0.86 & 0.61 & 1.20 & 0.80 & 0.73 & 0.88 & -0.06\end{array}$

$\begin{array}{lllllllll}\text { Michoacán } & 0.6 & 0.39 & 0.91 & 1.00 & 0.86 & 1.17 & +0.40\end{array}$

$\begin{array}{llllllll}\text { Morelos } & 0.67 & 0.49 & 0.91 & 0.89 & 0.70 & 1.14 & +0.22\end{array}$

$\begin{array}{lllllllll}\text { Nayarit } & 0.9 & 0.67 & 1.20 & 1.06 & 0.78 & 1.44 & +0.16\end{array}$

$\begin{array}{llllllll}\text { Puebla } & 0.61 & 0.41 & 0.88 & 0.67 & 0.57 & 0.79 & +0.06\end{array}$

$\begin{array}{lllllllll}\text { Querétaro } & 0.6 & 0.43 & 0.85 & 1.10 & 0.87 & 1.38 & +0.50\end{array}$

$\begin{array}{llllllll}\text { Tlaxcala } & 1.07 & 0.76 & 1.50 & 0.63 & 0.44 & 0.91 & -0.44\end{array}$

$\begin{array}{llllllll}\text { Región Sur } & \mathbf{0 . 8 5} & 0.78 & 0.93 & 0.74 & 0.68 & 0.8 & \mathbf{- 0 . 1}\end{array}$

\begin{tabular}{lllllllll}
\hline Campeche & 0.38 & 0.19 & 0.75 & 0.96 & 0.67 & 1.37 & +0.58 \\
\hline Chiapas & 1.16 & 0.82 & 1.64 & 0.55 & 0.44 & 0.68 & -0.61 \\
\hline Guerrero & 0.55 & 0.37 & 0.81 & 0.69 & 0.55 & 0.85 & +0.14 \\
\hline Oaxaca & 1.29 & 0.94 & 1.77 & 0.72 & 0.59 & 0.89 & -0.57 \\
\hline Quintana Roo & 0.67 & 0.43 & 1.04 & 0.43 & 0.28 & 0.65 & -0.24 \\
\hline Tabasco & 1.2 & 0.85 & 1.70 & 0.61 & 0.46 & 0.81 & -0.59 \\
\hline Veracruz & 0.43 & 0.24 & 0.76 & 0.99 & 0.87 & 1.11 & +0.56 \\
\hline Yucatán & 0.9 & 0.65 & 1.24 & 0.59 & 0.44 & 0.78 & -0.31 \\
\hline & & & & & & & +0.00 \\
\hline Distrito Federal & 1.44 & 1.07 & 1.95 & 1.44 & 1.32 & 1.56 & $+\mathbf{+ 0 . 0 0}$
\end{tabular}

Fuente: Estadísticas Vitales, Registros de Mortalidad, INEGI/SSA, 1979 a 2006, Proyecciones de población de Conapo 1970-2030. Referencia: Promedio Nacional
2006. Esta medida permite conocer dónde es mayor el riesgo de morir por esta causa, según sea el lugar de residencia urbano-rural en cada estado, en comparación con el promedio nacional. En consecuencia, esta medida sirve de complemento a las tasas observadas y estandarizadas de mortalidad porque hace posible observar con más detalle el comportamiento del indicador de acuerdo con el área de residencia. En cuanto al riesgo relativo de morir por cáncer de mama, se encontró que el riesgo de fallecer por cáncer de mama es menor en mujeres que residen en el área rural en relación con las que residen en el área urbana, en tanto que sucede lo contrario con el cáncer cervical.

Al comparar los riesgos entre el año 2000 y 2006, se advierte que a nivel nacional el riesgo de fallecer por cáncer de mama en 2000 en el área urbana era 2.3 veces mayor respecto del área rural y éste ha disminuido a 1.9 veces para el año 2006. En este punto, el riesgo de fallecer por cáncer de mama es $44 \%$ mayor en el área urbana en comparación con el área rural en el Distrito Federal, en relación con el promedio nacional, seguido por la región norte donde se observa un riesgo $14 \%$ mayor, mientras que en las regiones centro (0.94) y sur (0.74) el riesgo de fallecer por cáncer de mama es mayor en el área rural respecto del área urbana (cuadro I).

Se observó que en los estados que componen la región norte, sólo en Zacatecas (-0.06) se redujo el riesgo de fallecer por cáncer de mama en el 2006, en comparación con el riesgo presentado en el 2000. En los estados de la región centro disminuyó el riesgo en dos estados: Aguascalientes (-0.15) y Tlaxcala (-0.44), en tanto que en la región sur cinco estados redujeron el riesgo de morir por cáncer de mama en el área urbana respecto del área rural, lo cual indica que el riesgo de morir por cáncer de mama en el área rural ha aumentado y ha igualado el riesgo de morir por cáncer de mama en el área urbana; éstos son: Chiapas (-0.61), Oaxaca (-0.57), Quintana Roo $(-0.24)$, Tabasco (-0.59) y Yucatán (-0.31), como se observa en el cuadro I y la figura 3.

En el cuadro II se presentan los riesgos relativos de mortalidad por cáncer cervical según estrato urbanorural y entidad federativa para los años 2000 y 2006, los cuales delinean un comportamiento contrario al del cáncer de mama, como se mencionó. Mediante los riesgos relativos, se observa que en el año 2000 existe en el plano nacional un riesgo $23 \%$ mayor de morir por cáncer cervical en las mujeres que residen en el área rural respecto de las mujeres que lo hacen en el área urbana. Esto cambia para el año 2006, al registrarse en ese año tan sólo un riesgo 6\% mayor en el área rural en comparación con la urbana. A nivel regional, sólo en la región sur se 
Cuadro II

RIESGO DE MORIR POR CÁNCER CERVICAL POR ENTIDAD FEDERATIVA SEGÚN ESTRATO URBANO-RURAL. MÉxıco 2000 y 2006

\begin{tabular}{|c|c|c|c|c|c|c|c|}
\hline Entidad & IRR & $\begin{array}{c}2000 \\
\text { IC } \\
95 \%\end{array}$ & & IRR & $\begin{array}{l}2006 \\
\text { IC } \\
95 \%\end{array}$ & & $\begin{array}{l}\text { Diferencias } \\
\text { IRR de Cacu } \\
2000-2006\end{array}$ \\
\hline Área (Nacional) & 1.23 & 1.17 & 1.30 & 1.06 & 1.00 & I.II & +0.17 \\
\hline Región Norte & 0.92 & 0.86 & 0.99 & 0.93 & 0.87 & 1.00 & -0.01 \\
\hline Baja California & 0.93 & 0.77 & 1.12 & 0.72 & 0.58 & 0.90 & +0.21 \\
\hline Baja California Sur & 1.08 & 0.70 & 1.65 & 1.06 & 0.69 & 1.63 & +0.01 \\
\hline Coahuila & 0.94 & 0.77 & 1.14 & 0.95 & 0.78 & 1.17 & -0.02 \\
\hline Chihuahua & 1.04 & 0.88 & 1.22 & 0.93 & 0.78 & 1.12 & +0.11 \\
\hline Durango & 1.05 & 0.83 & 1.33 & 0.79 & 0.59 & 1.06 & +0.26 \\
\hline Nuevo León & 0.66 & 0.55 & 0.79 & 0.77 & 0.65 & 0.92 & -0.11 \\
\hline San Luis Potosí & 1.13 & 0.94 & 1.36 & 1.02 & 0.84 & 1.25 & +0.11 \\
\hline Sinaloa & 1.01 & 0.84 & 1.21 & 0.80 & 0.64 & 0.99 & +0.21 \\
\hline Sonora & 0.84 & 0.68 & 1.04 & 1.17 & 0.97 & 1.42 & -0.34 \\
\hline Tamaulipas & 1.00 & 0.84 & 1.19 & 1.20 & 1.02 & 1.42 & -0.20 \\
\hline Zacatecas & 0.68 & 0.50 & 0.92 & 0.94 & 0.71 & 1.24 & -0.26 \\
\hline
\end{tabular}

$\begin{array}{lllllllll}\text { Aguascalientes } & 0.77 & 0.55 & 1.07 & 0.69 & 0.48 & 0.99 & +0.07\end{array}$

\begin{tabular}{llllllll}
\hline Colima & 1.16 & 0.81 & 1.66 & 1.68 & 1.22 & 2.31 & -0.52
\end{tabular}

\begin{tabular}{llllllll}
\hline Guanajuato & 0.80 & 0.69 & 0.93 & 0.66 & 0.55 & 0.78 & +0.14
\end{tabular}

$\begin{array}{lllllllll}\text { Hidalgo } & 0.74 & 0.59 & 0.93 & 0.87 & 0.70 & 1.09 & -0.13\end{array}$

$\begin{array}{llllllll}\text { Jalisco } & \text { I.II } & 1.00 & 1.24 & 0.90 & 0.79 & 1.03 & +0.21\end{array}$

$\begin{array}{lllllllll}\text { México } & 0.78 & 0.71 & 0.86 & 0.86 & 0.78 & 0.94 & -0.08\end{array}$

\begin{tabular}{lllllllll}
\hline Michoacán & 1.17 & 1.02 & 1.34 & 1.07 & 0.92 & 1.25 & +0.09
\end{tabular}

$\begin{array}{lllllllll}\text { Morelos } & 1.64 & 1.38 & 1.96 & 1.55 & 1.27 & 1.88 & +0.10\end{array}$

$\begin{array}{lllllllll}\text { Nayarit } & 1.91 & 1.53 & 2.38 & 1.40 & 1.07 & 1.85 & +0.51\end{array}$

\begin{tabular}{lllllllll}
\hline Puebla & 0.98 & 0.86 & 1.11 & 1.14 & 1.00 & 1.30 & -0.16
\end{tabular}

\begin{tabular}{lllllllll}
\hline Querétaro & 0.80 & 0.61 & 1.05 & 0.79 & 0.60 & 1.04 & +0.01
\end{tabular}

$\begin{array}{lllllllll}\text { Tlaxcala } & 0.87 & 0.64 & 1.19 & 0.95 & 0.70 & 1.29 & -0.08\end{array}$

$\begin{array}{llllllll}\text { Campeche } & 1.26 & 0.92 & \text { I.71 } & \text { I.21 } & 0.87 & 1.68 & +0.05\end{array}$

$\begin{array}{lllllllll}\text { Chiapas } & 1.23 & 1.07 & 1.41 & 1.42 & 1.25 & 1.62 & -0.19\end{array}$

\begin{tabular}{lllllllll}
\hline Guerrero & 1.09 & 0.92 & 1.28 & 1.01 & 0.85 & 1.21 & +0.07
\end{tabular}

\begin{tabular}{lllllllll}
\hline Oaxaca & 1.12 & 0.96 & 1.31 & 1.15 & 0.98 & 1.35 & -0.03
\end{tabular}

$\begin{array}{lllllllll}\text { Quintana Roo } & 0.74 & 0.52 & 1.06 & 0.60 & 0.41 & 0.88 & +0.14\end{array}$

\begin{tabular}{lllllllll}
\hline Tabasco & 1.06 & 0.86 & 1.31 & 1.04 & 0.83 & 1.29 & +0.02
\end{tabular}

$\begin{array}{lllllllll}\text { Veracruz } & 1.34 & 1.22 & 1.48 & 1.43 & 1.29 & 1.58 & -0.08\end{array}$

$\begin{array}{lllllllll}\text { Yucatán } & 1.54 & 1.29 & 1.84 & 1.14 & 0.91 & 1.41 & +0.40\end{array}$

$\begin{array}{llllllll}\text { Distrito Federal } & 0.89 & 0.80 & 0.99 & 0.90 & 0.81 & 1.01 & -0.01\end{array}$

Fuente: EstadísticasVitales, Registros de Mortalidad, INEGI/SSA, 2000 a 2006, Proyecciones de población de CONAPO 1970-2030

Referencia: Promedio Nacional. * Los riesgos relativos fueron estimados en comparación con la residencia en localidades en el área urbana $(>2$ 500 habitantes) registra un mayor riesgo de morir por cáncer cervical entre las mujeres que residen en el área rural (22\% mayor), mientras que en las demás regiones vivir en área rural constituye un riesgo ligeramente menor.

En la figura 4 se observa que la región sur posee un riesgo 23\% mayor, respecto del promedio nacional, de morir por cáncer cervical en el área rural en comparación con el área urbana para el 2006, en tanto que las otras regiones poseen riesgos muy similares de fallecer por área de residencia urbana-rural con valores muy cercanos a 1. Es decir, el riesgo de morir por cáncer de mama ha aumentado en el área rural, pero el riesgo de morir por cáncer cervicouterino ha decrecido e igualado al riesgo de morir en el área urbana.

\section{Tasas de mortalidad por cáncer de mama y cervical por estado y grado de marginación}

En la figura 5 se presentan las tasas de cáncer de mama estandarizadas por entidad federativa para los años 2000 y 2006. Se observa que sólo seis de las 32 entidades mexicanas evidencian una disminución de las tasas estandarizadas de mortalidad por cáncer mamario entre los dos años de comparación; resaltan Nuevo León (-0.96), Zacatecas (-1.47), Yucatán (-1,21) y Aguascalientes (-1.05). Por el contrario, en los demás estados han aumentado las tasas de mortalidad por cáncer de mama, incluso en los estados más marginados.

$\mathrm{Al}$ relacionar las tasas de mortalidad por cáncer de mama con el grado de marginación de las entidades, se reconoce una leve correlación inversa, sobre todo en los grados extremos (muy alta o muy baja) de marginación. Esto es, en los estados con marginación muy alta, como Oaxaca, Chiapas y Guerrero, se encuentran tasas de mortalidad por cáncer de mama bajas, pero en los estados con muy bajo grado de marginación, como Coahuila, Baja California, Nuevo León y el Distrito Federal, sobresalen tasas elevadas de mortalidad por esta causa. Por el contrario, en los estados con niveles medios (alto, medio o bajo grado) de marginación, la tasa de mortalidad fluctúa en grado considerable y se encuentran estados con bajas y altas tasas de mortalidad por cáncer de mama, sin una tendencia lineal (figura 5).

En cuanto a la mortalidad por cáncer cervical por entidad federativa, se observa una tendencia decreciente en las cifras entre 2000 y 2006 (figura 6). Sin embargo, esta situación no es homogénea en todos los estados. Mientras que algunas entidades federativas experimenta disminuciones significativas en las tasas de mortalidad por esta causa, como son los estados de Nayarit (-8.4), Yucatán (-6.9), Morelos (-5.6) y Durango (-5.7), en otros 

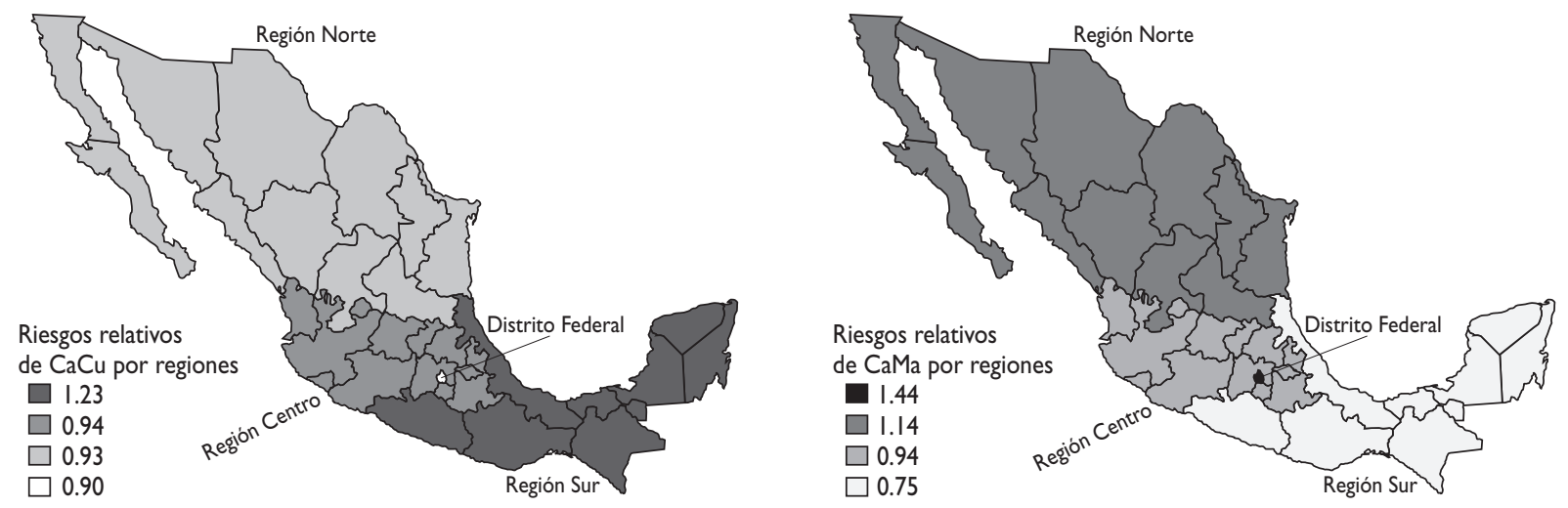

Fuente: estadísticas vitales del INEGI/SSA, México 2006 y las proyecciones de población 1970-2030, Conapo

Figura 4. Mapas Regionales de Riesgos relativos de mortalidad por CÁncer de mama y Cáncer Cervical. Méxıco 2006

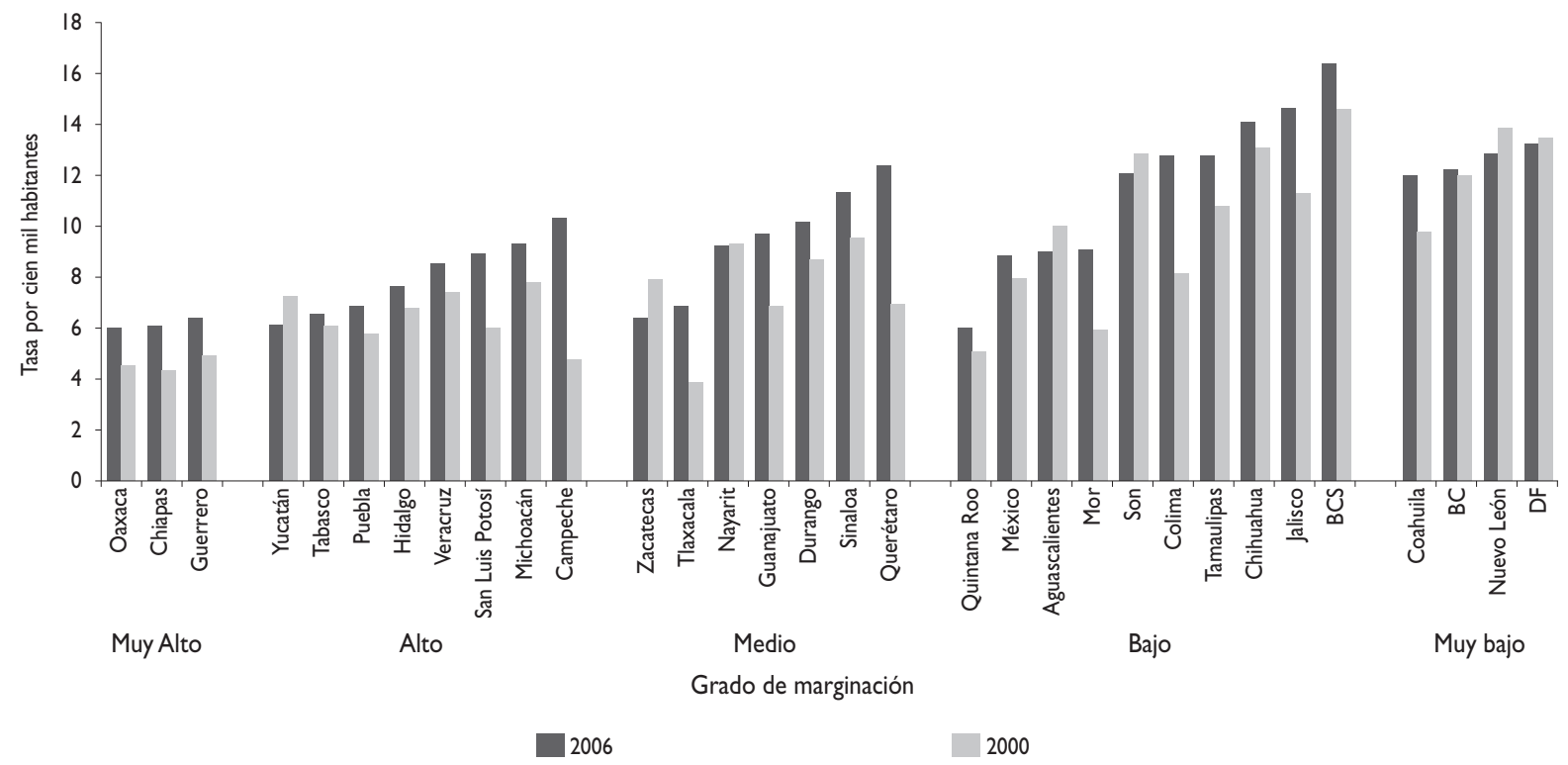

Fuente: elaboración a partir de las estadísticas vitales, registros de mortalidad, INEGI/SSA, 1979 a 2006, proyecciones de población de Conapo $1970-2030$ e índices de marginación 2005. Conapo

FiguRA 5.TASAS ESTANDARIZADAS DE MORTALIDAD POR CÁNCER DE MAMA POR ENTIDAD FEDERATIVA, SEGÚN grado de marginación. México 2000 y 2006

estados se identifican leves aumentos de las cifras, como Sonora (0.70), Zacatecas (0.85) y Colima (1.00). Las entidades con tasas estandarizadas de mortalidad por cáncer cervical más altas en 2006 son Chiapas (16.8), Colima (14.9), Morelos (13.8), Campeche (12.9), Vera- cruz (12.3), Baja California Sur (12.3) y Nayarit (12.3) y las entidades con menores tasas son Guanajuato (6.7), Nuevo León (6.9) y Distrito Federal (6.9).

Las tasas de mortalidad por cáncer cervical para el año 2006 de las entidades con un nivel muy bajo de mar- 


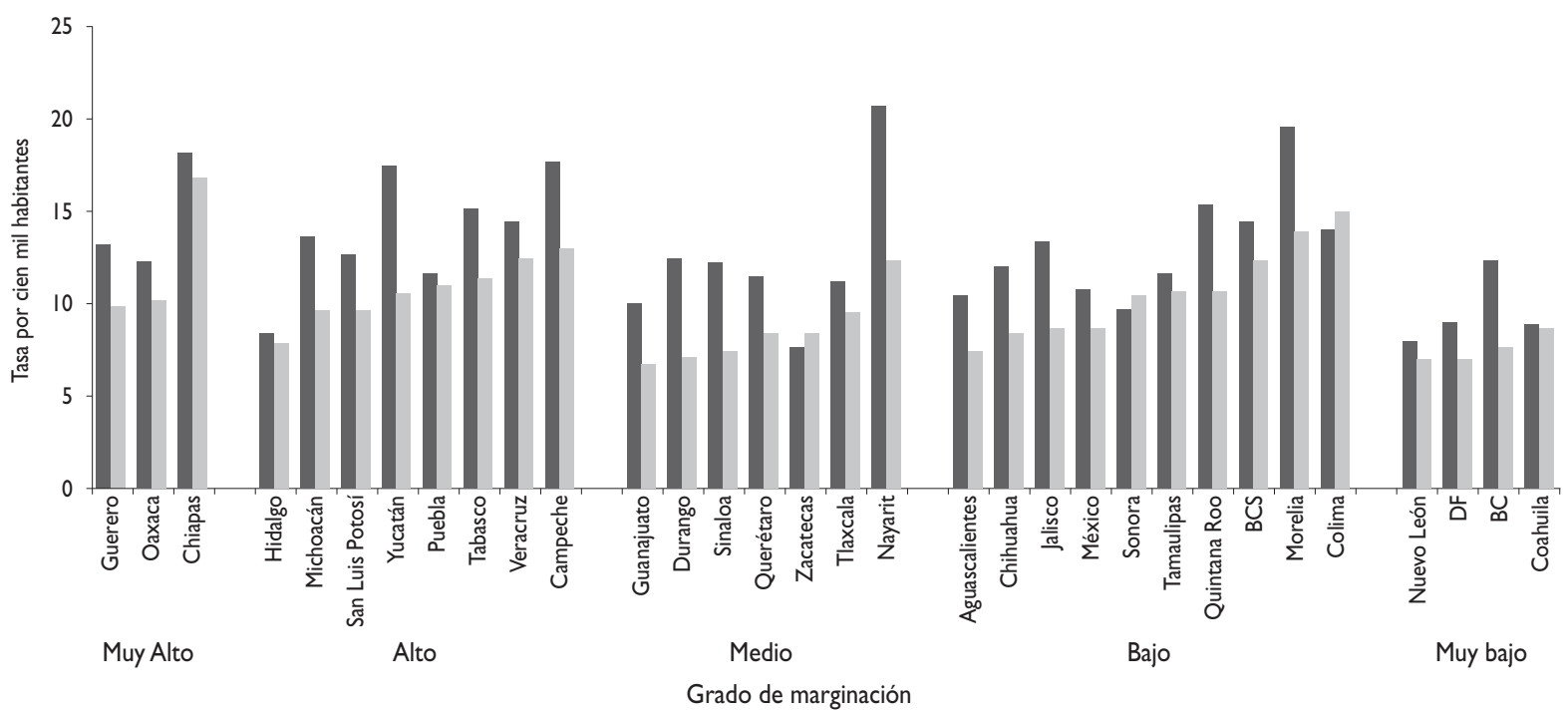

2006

2000

Fuente: elaboración a partir de las estadísticas vitales, registros de mortalidad, INEGI/SSA, 1979 a 2006, proyecciones de población de Conapo I970-2030 e índices de marginación 2005. Conapo.

FiguRA 6. TASAS ESTANDARIZADAS DE MORTALIDAD POR CÁNCER CERVICAL POR ENTIDAD FEDERATIVA, SEGÚN GRADO DE MARGINACIÓN. MÉXICO 2000 Y 2006

ginación muestran homogeneidad entre sí y Nuevo León y el Distrito Federal son las entidades que sobresalen por poseer la tasa más baja (6.9) (figura 6). En el caso de los estados con grado bajo de marginación, la situación es más heterogénea y sobresale Aguascalientes por tener la tasa más baja (7.4) y Colima la más alta (14.9). Esta misma heterogeneidad se reconoce en los estados con un grado de marginación medio y alto. Asimismo, en los estados con muy alto nivel de marginación, las tasas estandarizadas de Guerrero (9.8) y Oaxaca acusaron disminuciones considerables, con Chiapas (16.8) con la tasa más alta de mortalidad (figura 6).

\section{Discusión}

Los cánceres de mama y cervical representan las dos primeras causas de mortalidad por tumores malignos de mujeres en México. La evidencia que se presenta en este artículo ha permitido observar cómo siguen presentes las disparidades regionales en cuanto a la mortalidad por cáncer en la mujer mexicana. Aun cuando el número de muertes por cáncer de mama se incrementa de forma gradual a lo largo del tiempo a nivel nacional, en áreas marginadas este aumento es notablemente significativo, en el mismo ámbito donde persisten elevadas tasas de mortalidad por cáncer cervical. Los hallazgos señalan que mientras la tendencia de mortalidad por cáncer de mama ha sido ascendente en el plano nacional durante el periodo de 1979 a 2006, la mortalidad por cáncer cervical ha disminuido de forma notoria desde 1990.

A partir del año 2006, el cáncer de mama se vuelve la primera causa de mortalidad por cáncer en mujeres en México. A nivel regional, el cáncer de mama tiene tendencias ascendentes y es mayor en el norte del país y el Distrito Federal. Al comparar el riesgo de morir por cáncer de mama en 2000 y 2006, el riesgo de fallecer por esta causa se redujo de 2.33 veces más para mujeres con residencia urbana a 1.88 veces más, lo cual implica que mientras el riesgo para mujeres en áreas urbanas disminuye, en mujeres en áreas rurales aumenta. La tendencia ascendente generalizada de la mortalidad por cáncer de mama podría deberse a la virtual ausencia de infraestructura para el tamizaje y, en consecuencia, la imposibilidad de reconocer de manera oportuna esta neoplasia en México. Por su parte, la mortalidad por cáncer cervical es significativamente mayor en la región sur, donde existe un predominio de áreas marginadas, lo que tal vez indique que la accesibilidad geográfica y cultural a los servicios de detección oportuna sea menor, al tiempo que podría haber menor penetración de la educación para la salud sobre este tema. ${ }^{23,24} \mathrm{La}$ pobreza, cuando se mide a través de un índice de 
marginación de la región donde viven las mujeres, se vincula con un limitado acceso a la atención primaria a la salud, así como escasas accesibilidad y calidad de los programas de prevención poblacional que utilizan pruebas de tamizaje, lo cual tiene como resultado muertes por causas potencialmente prevenibles. ${ }^{25-27}$ Por otra parte, diversos factores culturales y de injusticia social, expresados en la falta de percepción de riesgo de enfermedad y experiencias previas de despersonalización de los servicios de atención médica, han sido causas tradicionales de disparidad en salud y del incremento del número de muertes por cáncer en la mujer en la población marginada, ${ }^{28-35}$ como el presente análisis evidencia en mujeres mexicanas.

En este artículo se exploraron las disparidades en la mortalidad por estas dos neoplasias al correlacionar tasas de mortalidad en función del grado de marginación en México desde la perspectiva de un modelo ecológico. En el caso del cáncer de mama, en estados con marginación muy alta (como Oaxaca, Chiapas y Guerrero) hay tasas bajas de mortalidad por cáncer de mama y en estados con marginación muy baja hay altas tasas de mortalidad por esta tumoración (Baja California, Coahuila, Nuevo León y el Distrito Federal). En el caso del cáncer cervical se observa lo contrario, ya que en estados con un índice muy bajo de marginación se reconocen las tasas más bajas por esta causa (Distrito Federal y Nuevo León) y en las entidades federativas con el índice de marginación más alto se identifican las tasas más altas de mortalidad por cáncer cervical (Chiapas, Oaxaca y Guerrero). No obstante, en las regiones con elevada marginación coexisten en la actualidad un elevado número de muertes por cáncer de mama y de muertes por cáncer cervical.

Es indispensable la investigación sobre las disparidades descritas en relación con el cáncer de mama y cervical en México, así como de otras neoplasias, para elaborar y poner en práctica medidas de prevención y control basadas en evidencia científica. ${ }^{7,15,36,37}$ Se han descrito determinantes sociales de disparidad en salud en relación con la mortalidad por cáncer de mama y cervical, en diversos grupos étnicos y niveles socioeconómico y educativo, casi exclusivamente en países desarrollados. ${ }^{3,4,5,12-14,38}$ Se han señalado diversas barreras sociales que tienen un efecto en la expresión biológica de la enfermedad, en función de una menor sobrevida relacionada con diversos fenómenos, entre ellos un diagnóstico tardío. ${ }^{7,12,13,39}$ Hoy en día se han propuesto diversas alternativas creativas para promover el acceso a la detección del cáncer de mama en áreas geográficas sin infraestructura de detección oportuna de cáncer. ${ }^{40,41}$ Un ejemplo de ello es la descentralización de programas de prevención y control en el ámbito comunitario, que se ha intentado en diversos países para incrementar la cobertura de tamizaje de cáncer en la mujer en áreas marginadas. ${ }^{42} \mathrm{~A}$ este respecto, existen reportes previos que establecen el impacto de una menor infraestructura de tratamiento en la terapéutica y la mortalidad por cáncer de mama entre mujeres que habitan en el ámbito rural, ${ }^{43}$ lo cual podría constituir una parte importante de la problemática en México. En este sentido, programas dirigidos a mujeres de bajos recursos en áreas marginadas, siempre que cuenten con suficiente infraestructura y personal de salud, pueden atenuar las disparidades en la detección y tratamiento del cáncer de mama y cervical. ${ }^{44}$

La bibliografía sobre las inequidades en las tendencias de mortalidad por cáncer cervical y de mama muestra que el riesgo de morir por dichas neoplasias se vincula inversamente con el nivel socioeconómico y diversos indicadores de marginación, tales como grupo ético, residencia rural y otros. ${ }^{7,12,13,26,28,29,36,45}$ A este respecto, se ha documentado que mujeres con mayores ingresos, que tienen el mismo estadio de enfermedad y condición mórbida, muestran una sobrevida mayor en comparación con las mujeres de menor estrato social. ${ }^{46}$ Asimismo, se ha documentado ampliamente que el lugar de residencia constituye un factor de riesgo para el desarrollo de cáncer de mama ${ }^{47}$ y cervicali; ${ }^{13,26}$ estudios ecológicos como éste en relación con el panorama de mortalidad por cáncer en México son también una nueva evidencia de ello. Estos hechos son significativos, ya que es necesario considerar que no sólo es preciso poner en práctica acciones de salud pública, sino diseñar tales acciones de tal manera que se reduzcan las inequidades en el acceso y uso de servicios que puedan contribuir a abatir el riesgo de morir por enfermedades prevenibles o tratables. ${ }^{7,12,13,36,40,41,44}$

Por último, es posible concluir que para coadyuvar a disminuir las elevadas tasas de mortalidad por cáncer de mama y cervical en mujeres mexicanas es necesario instituir diversas medidas con una perspectiva de innovación y equidad. Entre dichas disposiciones figuran crear campañas de educación para la salud que empoderen a las mujeres en relación con estas neoplasias, incrementar la cobertura de la detección, facilitar el acceso a la detección para mujeres con mayores barreras geográficas, culturales o económicas, instalar mecanismos de control de calidad para la confirmación diagnóstica, aplicar tecnologías y campañas preventivas (como la vacuna contra el virus del papiloma humano para el cáncer cervical) y garantizar el tratamiento completo y de similar calidad para todas las mujeres. ${ }^{48-52}$ En conjunto, se trata de dar una respuesta social organizada a un enorme desafío que permanentemente será necesario enfrentar: la desigualdad social. 


\section{Referencias}

I. Goddard M, Smith P. Equity of access to health care services: theory and evidence from the UK. Soc Sci Med 200 I;53(9): I I49-I I 62.

2. Beccaro M, Costantini M, Merlo DF, ISDOC Study Group. Inequity in the provision of and access to palliative care for cancer patients. Results from the Italian survey of the dying of cancer (ISDOC). BMC Public Health 2007;7:66.

3.Ward E, Jemal A, Cokkinides V, Singh GK, Cardinez C, Ghafoor A, et al. Cancer disparities by race/ethnicity and socioeconomic status. CA Cancer J Clin 2004;54(2):78-93.

4. Freeman HP, Chu KC. Determinants of cancer disparities: barriers to cancer screening, diagnosis, and treatment. Surg Oncol Clin N Am 2005; |4(4):655-669.

5. Kolb B,Wallace AM, Hill D, Royce M. Disparities in cancer care among racial and ethnic minorities. Oncology 2006;20(I0): I256-1270.

6. Qazi S, DuMez D, Uckun FM. Meta analysis of advanced cancer survival data using lognormal parametric fitting: a statistical method to identify effective treatment protocols. Curr Pharm Des 2007; |3(I5):I533-I544. 7. Krieger N. Defining and investigating social disparities in cancer: critical issues. Cancer Causes Control 2005; 16(I):5-14.

8. Hairon N. Report finds inequity in access to breast surgery services. Nurs Times 2008; 104(II):21-22.

9. Tovar-Guzman V, Hernandez-Giron C, Lazcano-Ponce E, Romieu I, Hernandez Avila M. Breast cancer in Mexican women: an epidemiological study with cervical cancer control. Rev Saude Publica 2000;34(2): I 3-I I 9 10. Couture MC, Nguyen CT,Alvarado BE, Velasquez LD, Zunzunegui MV. Inequalities in breast and cervical cancer screening among urban Mexican women. Prev Med 2008;47(5):47I-476.

I I. Lazcano-Ponce E, Palacio-Mejia LS,Allen-Leigh B,Yunes-Diaz E,Alonso P, Schiavon R, Hernandez-Avila M. Decreasing cervical cancer mortality in Mexico: effect of Papanicolaou coverage, birthrate, and the importance of diagnostic validity of cytology. Cancer Epidemiol Biomarkers Prev 2008; 17(10):2808-28I7.

12. Bigby J, Holmes MD. Disparities across the breast cancer continuum. Cancer Causes Control 2005; I6(I):35-44.

13. Bradley CJ, Given CW, Roberts C. Health care disparities and cervical cancer.Am J Public Health 2004;94(I2):2098-2103.

14.Aziz Z, lqbal J,Akram M; FCPS. Effect of social class disparities on disease stage, quality of treatment and survival outcomes in breast cancer patients from developing countries. Breast J 2008; I4(4):372-375.

15. Koh HK, Judge CM, Ferrer B, Gershman ST. Using public health data systems to understand and eliminate cancer disparities. Cancer Causes Control 2005; I6(I):15-26.

16. Palacio-Mejia LS, Rangel-Gomez G, Hernandez-Avila M, Lazcano-Ponce E. Cervical cancer, a disease of poverty: mortality differences between urban and rural areas in Mexico. Salud Publica Mex 2003;45(Suppl 3):S3I5-S325. 17. Secretaría de Salud de México. Sistema Nacional de Información en Salud SINAIS. Cubos Dinámicos de Mortalidad 1979 a 2006. Disponible en: www.sinais.salud.gob.mx

18. Consejo Nacional de Población CONAPO (2006) Proyecciones de Población 1970 a 2030 por entidad federativa. Disponible en: www. conapo.gob.mx

19. Naciones Unidas. División de Población del Departamento de Asuntos Sociales y Económicos de la Secretaría de Naciones Unidas, publicadas en el documento Perspectivas de la Población Mundial: Revisión 2004 y Perspectivas de la Urbanización Mundial: Revisión 2003. Disponible en: http://esa.un.org/unpp, febrero 2006.

20. Gobierno de la República de México 2007 Plan Nacional de Desarrollo 2007-2012, México. México: Gobierno Federal, 2007. 21. Consejo Nacional de Población CONAPO (2006). Índices de Marginación 2005. Disponible en: http://www.conapo.gob.mx/ publicaciones/margina2005/0I_b.pdf.
22. Instituto Nacional de Estadística, Geografía en Informática INEGI. Catálogos y clasificadores geográficos. Disponible en: www.inegi.gob.mx 23. Buki LP, Jamison J,Anderson CJ, Cuadra AM. Differences in predictors of cervical and breast cancer screening by screening need in uninsured Latino women. Cancer 2007; I 10(7):1578-1585.

24. Rogers NM, Cantu AG. The Nurse's role in the prevention of cervical cancer among underserved and minority populations. J Community Health 2008 Oct 24. [Epub ahead of print]

25. Westin SN, Bustillos D, Gano JB, Fields MM, Coker AL, Sun CC, et al. Social factors affecting treatment of cervical cancer: ethical issues and policy implications. Obstet Gynecol 2008; I I (3):747-75 I.

26. Yabroff KR, Lawrence WF, King JC, Mangan P,Washington KS, Yi B, et al. Geographic disparities in cervical cancer mortality: what are the roles of risk factor prevalence, screening, and use of recommended treatment? J Rural Health 2005;2I (2): 149-I57.

27. Datta GD, Colditz GA, Kawachi I, Subramanian SV, Palmer JR, Rosenberg L. Individual-, neighborhood-, and state-level socioeconomic predictors of cervical carcinoma screening among U.S. black women: a multilevel analysis. Cancer 2006; 106(3):664-669.

28. Gerend MA, Pai M. Social determinants of black-white disparities in breast cancer mortality: a review. Cancer Epidemiol Biomarkers Prev 2008; 17(II):2913-2923.

29. Bailie RS, Selvey CE, Bourne D, Bradshaw D. Trends in cervical cancer mortality in South Africa. Int J Epidemiol 1996;25(3):488-493.

30. Dietsch E, Gibb H, Francis K.Abnormal Pap test results and the rurality factor. Aust J Rural Health 2003; I I (2):50-56.

3I. Friedell GH, Tucker TC, McManmon E, Moser M, Hernandez C, Nadel $\mathrm{M}$. Incidence of dysplasia and carcinoma of the uterine cervix in an Appalachian population.J Natl Cancer Inst 1992;84(I3): 1030- 1032. 32. Palacio-Mejia LS, Rangel-Gomez G, Hernandez-Avila M, Lazcano-Ponce E. Cervical cancer, a disease of poverty: mortality differences between urban and rural areas in Mexico. Salud Publica Mex 2003;45(Suppl 3): S3I5-S325.

33. Binns PL, Condon JR. Participation in cervical screening by Indigenous women in the Northern Territory: a longitudinal study. Med J Aust 2006; 185(9):490-494.

34. Wain G, Morrell S, Taylor R, Mamoon H, Bodkin N.Variation in cervical cancer screening by region, socio-economic, migrant and indigenous status in women in New South Wales. Aust N Z J Obstet Gynaecol 200I;4I(3):320-325.

35. Coory MD, Fagan PS, Muller JM, Dunn NA. Participation in cervical cancer screening by women in rural and remote Aboriginal and Torres Strait Islander communities in Queensland. Med J Aust 2002;177(10): 544-547.

36. Newmann SJ, Garner EO. Social inequities along the cervical cancer continuum: a structured review. Cancer Causes Control 2005; I6(I):63-70. 37. Woodward A, Kawachi I.Why reduce health inequalities? J Epidemiol Comm Health 2000;54(I2):923-929.

38. Clegg LX, Reichman ME, Miller BA, Hankey BF, Singh GK, Lin YD, et al. Impact of socioeconomic status on cancer incidence and stage at diagnosis: selected findings from the surveillance, epidemiology, and end results: National Longitudinal Mortality Study. Cancer Causes Control 2008 Nov 12. [Epub ahead of print]

39. Sabatino SA, Coates RJ, Uhler RJ, Breen N, Tangka F, Shaw KM. Disparities in mammography use among US women aged 40-64 years, by race, ethnicity, income, and health insurance status, 1993 and 2005. Med Care 2008;46(7):692-700

40. Erwin DO, Johnson VA, Trevino M, Duke K, Feliciano L, Jandorf L.A comparison of African American and Latina social networks as indicators for culturally tailoring a breast and cervical cancer education intervention. Cancer 2007;109(Suppl 2):368-377.

4I.Ashing-Giwa KT. Enhancing physical well-being and overall quality of life among underserved Latina-American cervical cancer survivors: feasibility study.J Cancer Surviv 2008;2(3):215-223. 
42. Bhaskara A,Altamirano M,Trisal V, Paz IB, Lai LL. Effectiveness of decentralized community-based screening, detection, and treatment of breast cancer in low-income, uninsured women. Am Surg 2008;74(10):1017-1021.

43. Esnaola NF, Knott K, Finney C, Gebregziabher M, Ford ME. Urban/rural residence moderates effect of race on receipt of surgery in patients with nonmetastatic breast cancer: a report from the South Carolina central cancer registry.Ann Surg Oncol 2008;15(7):1828-1836.

44. Liu MJ, Hawk H, Gershman ST, Smith SM, Karacek R, Woodford ML, et al. The effects of a National Breast and Cervical Cancer Early Detection Program on social disparities in breast cancer diagnosis and treatment in Massachusetts. Cancer Causes Control 2005; 16(I):27-33.

45. Chu KC, Miller BA, Springfield SA. Measures of racial/ethnic health disparities in cancer mortality rates and the influence of socioeconomic status.J Natl Med Assoc 2007;99(10): 1092-100, I 102-I 104.

46. Dalton SO, Ross L, Düring M, Carlsen K, Mortensen PB, Lynch J, et al. Influence of socioeconomic factors on survival after breast cancer--a nationwide cohort study of women diagnosed with breast cancer in Denmark 1983-1999. Int J Cancer 2007; I2I(II):2524-253 I.
47. MacKinnon JA, Duncan RC, Huang Y, Lee DJ, Fleming LE, Voti L, et al. Detecting an association between socioeconomic status and late stage breast cancer using spatial analysis and area-based measures. Cancer Epidemiol Biomar Prev 2007;16(4):756-762.

48. Zapka JG, Taplin SH, Solberg LI, Manos MM.A framework for improving the quality of cancer care: the case of breast and cervical cancer screening. Cancer Epidemiol Biomar Prev 2003; I2(I):4-I3.

49. Zapka JG, Lemon SC. Interventions for patients, providers, and health care organizations. Cancer 2004; I0I(Suppl 5): I 165-I I87.

50. Glasgow RE, Marcus AC, Bull SS, Wilson KM. Disseminating effective cancer screening interventions. Cancer 2004;10 I (Suppl 5): I239-1250.

5I. Sabatino SA, Habarta N, Baron RC, Coates RJ, Rimer BK, Kerner J, et al. Interventions to increase recommendation and delivery of screening for breast, cervical, and colorectal cancers by healthcare providers systematic reviews of provider assessment and feedback and provider incentives. Am J Prev Med 2008;35(Suppl I):S67-74.

52. Goins KV, Zapka JG, Geiger AM, Solberg LI, Taplin S, Yood MU, Gilbert J, Mouchawar J, Somkin CP,Weinmann S. Implementation of systems strategies for breast and cervical cancer screening services in health maintenance organizations. Am J Manag Care 2003;9(I I):745-755. 